Research Paper: Pathology

\title{
Novel mutations c.28G $>$ T (P.Ala10Ser) and c.189G $>$ T (p.Glu63Asp) in WDR62 associated with early onset acanthosis and hyperkeratosis in a patient with autosomal recessive microcephaly type 2
}

\author{
Santasree Banerjee ${ }^{1, *}$, Huishuang Chen ${ }^{1, *}$, Hui Huang ${ }^{1, *}$, Jing Wu ${ }^{1}$, Zhiyun Yang ${ }^{2}$, \\ Weiping Deng ${ }^{3}$, Dongna Chen ${ }^{1}$, Jianlian Deng ${ }^{1}$, Yan Su${ }^{1}$, Yang $\mathbf{L i}^{1}$, Chao Wu ${ }^{6}$, Ye \\ Wang ${ }^{4}$, Hao Zeng ${ }^{4}$, Yiming Wang ${ }^{5,1}$ and Xunhua Li ${ }^{6}$ \\ ${ }^{1}$ BGI-Shenzhen, Shenzhen, China \\ 2 Department of Medical Imaging, 1st affiliated hospital, Sun Yat-sen University, Guangzhou, PR China \\ ${ }^{3}$ Department of Dermatology, Guangdong General Hospital, Guangdong Academy of Medical Sciences, PR China \\ ${ }^{4}$ Department of Medical Genetics, Center for Genome Research, Sun Yat-sen University, Guangzhou, PR China \\ ${ }^{5}$ Xinhua College, Sun Yat-sen university, Guangzhou, PR China \\ ${ }^{6}$ Department of Neurology, 1st affiliated hospital, Sun Yat-sen University, Guangzhou, PR China \\ * These authors have contributed equally to this work \\ Correspondence to: Xunhua Li, email: Ixh59xyh@sina.com
}

Yiming Wang, email: ywzhong@hotmail.com

Keywords: microcephaly 2, WDR62 mutation, compound heterozygosity, novel mutations, exome sequencing, Pathology Section Received: June 25, 2016

Accepted: November 01, 2016

Published: November 10, 2016

\section{ABSTRACT}

Microcephaly (MCPH) is a developmental disorder characterized by reduced brain size and intellectual disability. A proportion of microcephaly is caused by defects in a single gene. Microcephaly 2 (MCPH2) is one of the most frequent subtypes of MCPH. WD repeat-containing protein 62 gene (WDR62) is the most frequently mutated gene in MCPH2 patients. Phenotypes involving dermatological changes in MCPH2 have not been reported. We have identified and investigated a 5-year-old Chinese girl with markedly reduced brain size ( $86 \%$ of normal size), intellectual disability and psychomotor developmental delay. The patient also exhibited spattered blisters and reduced hair density on her head, anisochromasia with reticular hyperpigmentation and hypopigmentation on the trunk, which she has had since the age of 4 and had been found by her parents. Histological examination of a skin biopsy revealed acanthosis, hyperkeratosis and necrotic keratinocytes. Whole exome and Sanger sequencing identified two novel missense mutations, c.28G $>T$ and $c .189 G>T$, in the WDR62 gene. Both the mutations non-synonymously affect evolutionarily conserved amino acids and are predicted to be disease causing. We report the first case of MCPH2 that also presented with marked dermatological changes. Our findings expand the mutational and phenotypical spectra of MCPH2 and are valuable in the mutation-based pre- and post-natal screening and genetic diagnosis for MCPH2.

\section{INTRODUCTION}

Microcephaly (MCPH) is a disorder of fetal brain growth; individuals with microcephaly have small brains and almost always have intellectual disability. The etiology of microcephaly can be hereditary or environmental, as with the Zika virus-related MCPH.
Hereditary microcephaly constitutes a large proportion of the cases, with 17 loci registered in the Online Mendelian Inheritance in Man database (MIM, omim. org/), and each is caused by a distinct gene. However, most subtypes of MCPH have overlapping phenotypes. Dermatological involvement in MCPH has only been reported in two patients, who had prenatal and postnatal 
growth retardation, microcephaly, developmental delay, generalized reticular hyperpigmentation, hypohidrosis, with absence of fingertip prints and palmoplantar hyperkeratosis [1]. However, the etiology of these two cases was unidentified.

Autosomal recessive primary microcephaly 2 (MCPH2) [MIM\#604317] has a prevalence between 1 in 30,000 and 1 in 250,000[2]. It is the second most common subtypes of MCPH [3]. The clinical diagnosis of $\mathrm{MCPH} 2$ is based on a head circumference more than 3 standard deviations (SD) below the age- and sex-matched population mean and mental retardation with exclusion of other causes [4]. The WD repeat-containing protein 62 (WDR62) gene has been identified as a genetic cause of MCPH2. Patients with WDR62 mutations present with a wide spectrum of cortical malformations including cortical thickening, polymicrogyria, abnormal/simplified gyral pattern, pachygyria, hypoplasia of the corpus callosum, and schizencephaly, heterotopias [5]. Some patients also have evidence of lissencephaly, cerebellar hypoplasia, and hippocampal dysmorphy $[6,7,8]$. A broad range of neurological and behavioral manifestations have been reported, including decreased fetal movements, delayed psychomotor development, mental retardation, impulsivity and aggression [9]. Phenotypes involving dermatological symptoms have not been reported in $\mathrm{MCPH} 2$. In this paper, we report our investigation of a Chinese girl who exhibited marked dermatological phenotypes and the associated two novel compound heterozygous mutations in WDR62.

\section{RESULTS}

\section{Clinical findings}

Proband is a 5-year-old girl, the first child of nonconsanguineous healthy parents of Han Chinese (Figure 1A), presented with psychomotor retardation since infancy. She was unable to raise her head and sit without external support until she was 3 years old. She could not walk independently. She could not say any words except "mom" and "dad." Feeding had been difficult due to frequent vomiting, constipation, anemia and recurrent infection of the respiratory tract. Hair was easily lost, resulting in scant hair on the top of the head. However, seizures had not been observed.

Our physical examinations showed that she was conscious but apathetic. She could not communicate verbally. Her height was $73 \mathrm{~cm}$, and her weight was 6 $\mathrm{kg}$. Her head circumference was only $43 \mathrm{~cm}$, which is $86 \%$ of the normal range. She was hypomyotonic and had scoliosis. She had facial features typical of MCPH2 (Figure 1B), which manifest in a wide and low bridge of the nose, thick lips, broad eye distance, sloping forehead, high palate-maxillary arch and low-set and large ears. Brain imaging showed a slight expansion of bilateral brain ventricles, which was apparent on the left side; obvious expansion of the fourth ventricle; thinning of the corpus callosum with the absence of the splenium; dysplasia of the temporal lobe with small hippocampus,
(A)

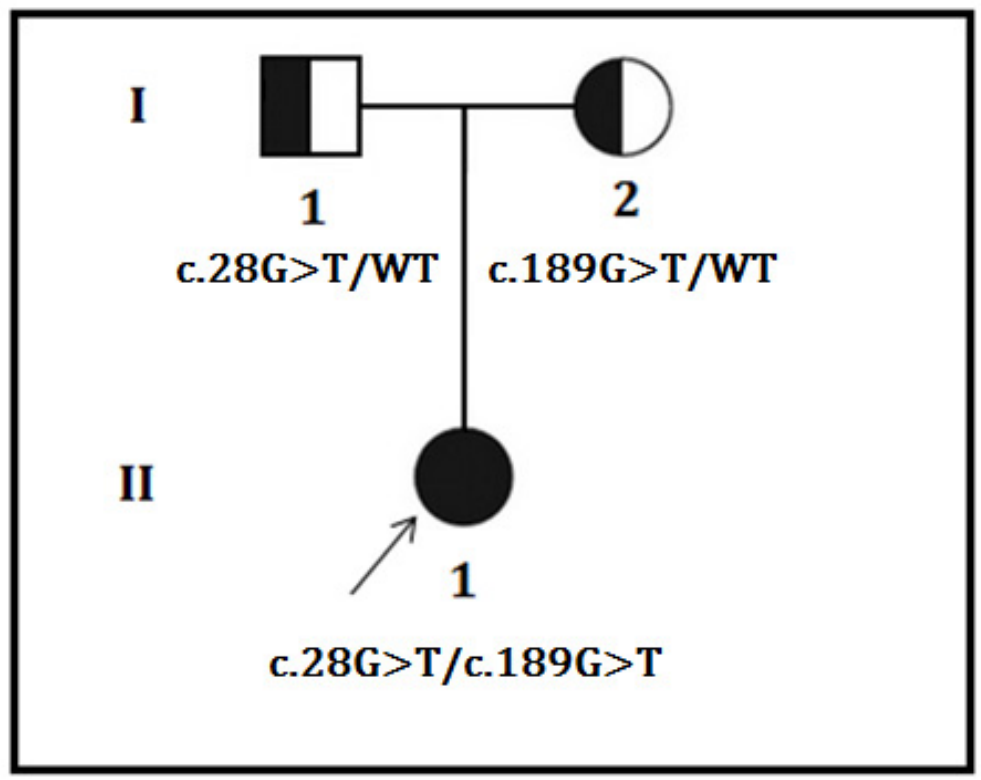

(B)

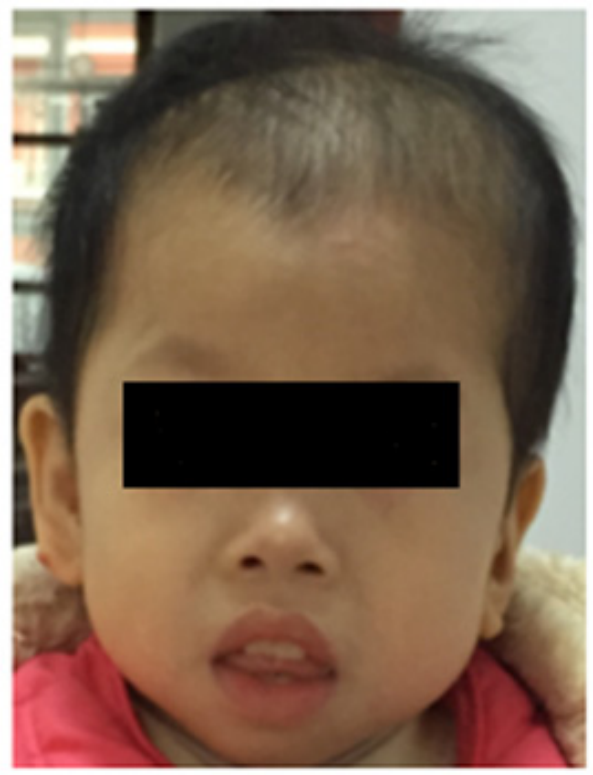

Figure 1: A. Pedigree of the family. The filled symbol indicates the patient (proband), and the half-filled symbols show the carrier parents, who were heterozygous carriers but were unaffected. The arrow points to the proband. B. Facial phenotype of the patient, which shows the sloping forehead, the convex facial profile, the full lips, and the small chin. The appearance of low-set and posteriorly rotated ears in the lateral picture is partly due to reclamation of the head. It also shows the reduced hair on the top of the head. 
enlarged temporal horn and broadening lateral fissure; leukodystrophy, dysplasia of the white matter; and suspected schizencephaly in the right parietal lobe and slight atrophy of the brain stem and cerebellum. (Figure $2 \mathrm{~A}-2 \mathrm{E})$. Abdominal ultrasound examinations showed that her liver measured $1.6 \mathrm{~cm}$ under the ribs. Her kidneys were $4 \times 2.2 \mathrm{~cm}$ on the right side and $3.8 \times 2.3 \mathrm{~cm}$ on the left side.

(A)

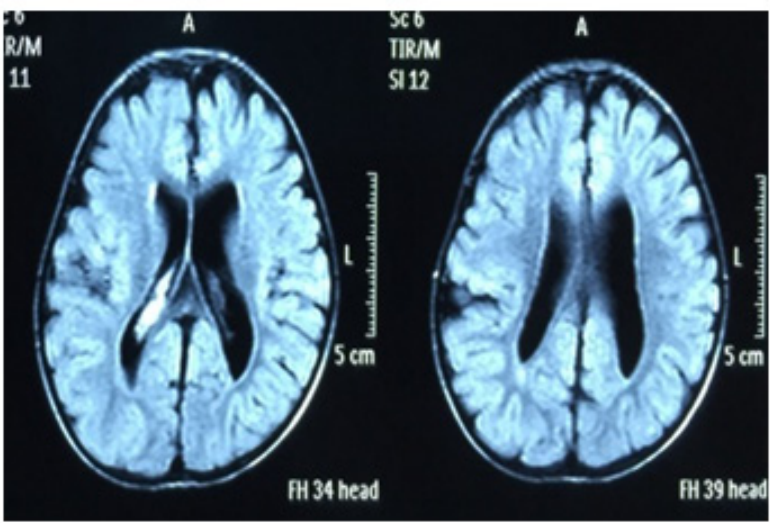

(C)

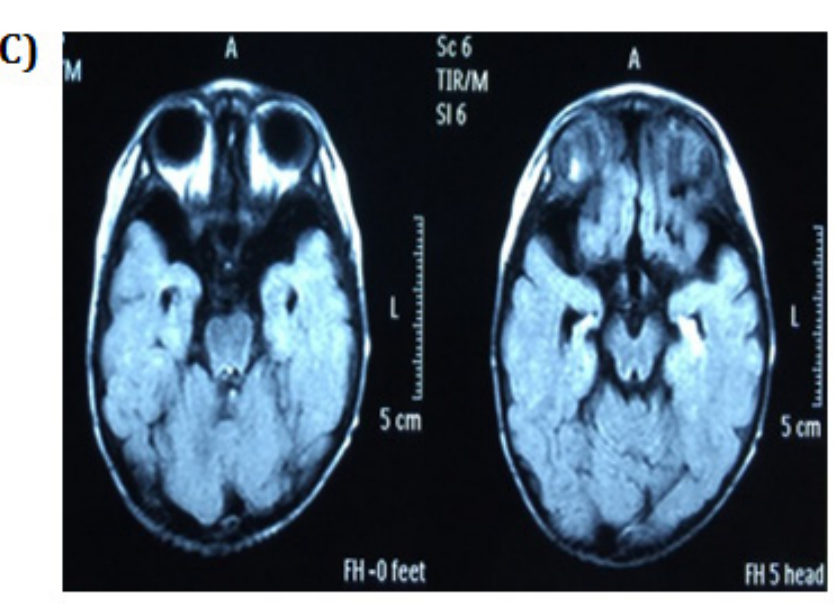

(D)

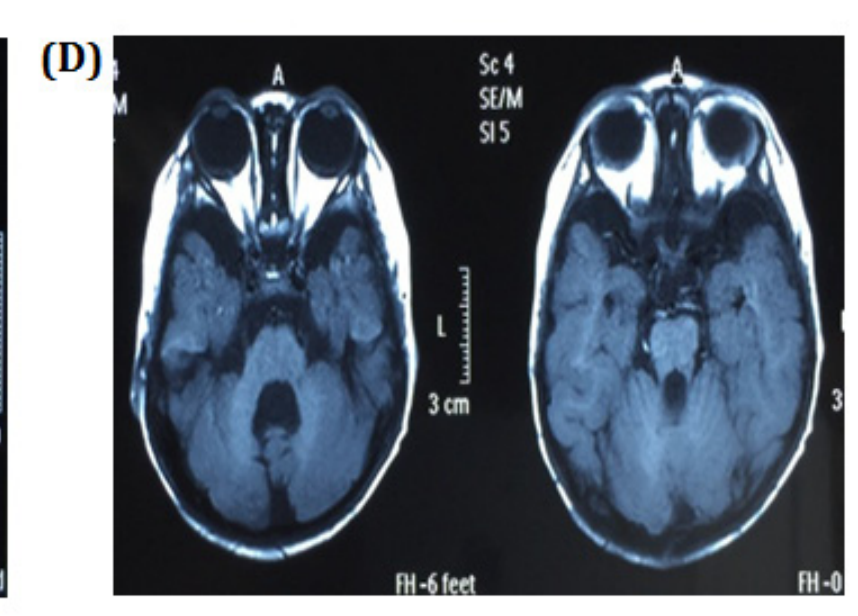

(B)

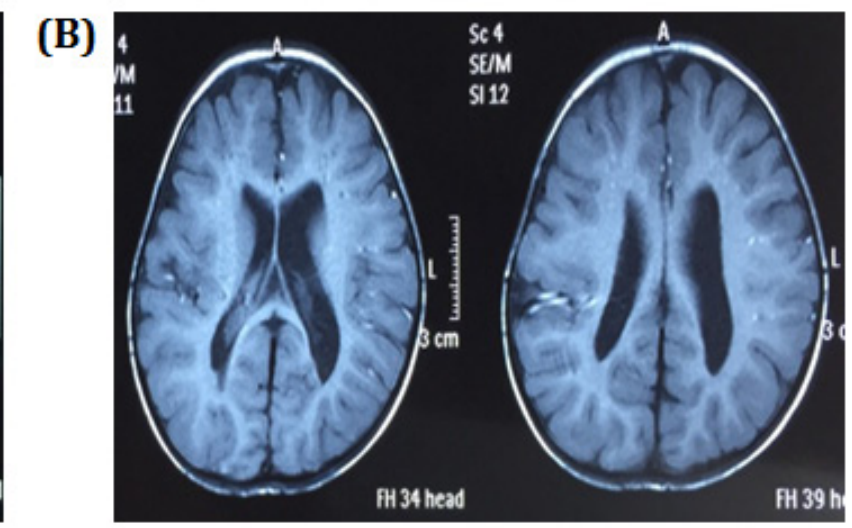
unusual skin lesions, the spattered blisters and the reduced hair density (Figure 3A) on her head and the anisochromasia with reticular hyperpigmentation and hypopigmentation on the trunk (Figure 3B, 3C) that had first been observed by her parents when she was four years old. Her nails and mucous membranes appeared normal. Histological examination of skin biopsy revealed

(E)

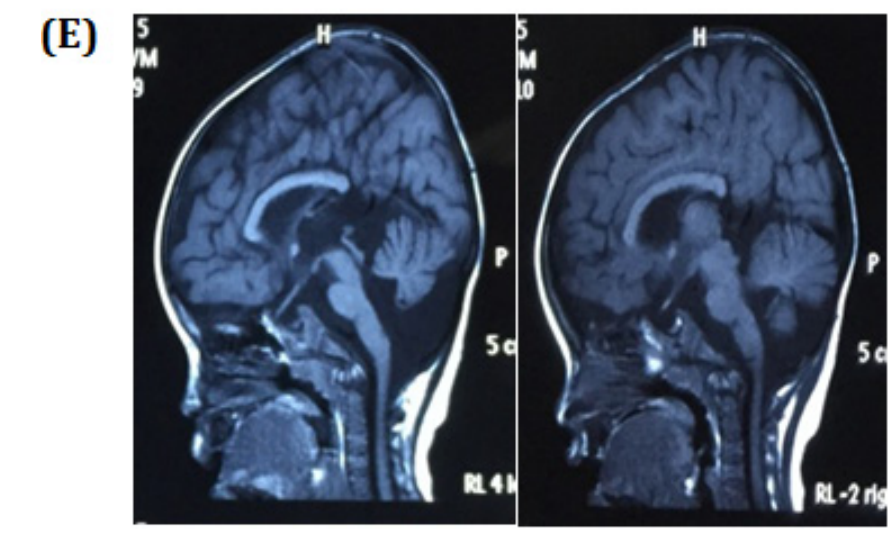

Figure 2: Brain images A. and B. showing the expansion of bilateral brain ventricles, dysplasia of the brain white matter and suspicious schizencephaly in the right parietal lobe, $\mathbf{C}$. dysplasia of the temporal lobe with small hippocampus and enlarge temporal horn, D. obvious expansion of the fourth ventricle and slight atrophy of cerebellum, and E. slight atrophy of the brain stem and thinning of the corpus callosum with absence of the splenium. 
acanthosis, hyperkeratosis and necrotic keratinocytes. There was melanin in melanophages in the upper dermis (Figure 3D, 3E). Based on the results of our clinical and genetic testing, we diagnosed her with MCPH2.

Written informed consent was obtained from the parents. The project was approved by the ethics committee of the BGI and in accordance with the Principles of the Declaration of Helsinki.

\section{Karyotyping and chromosomal array analysis excluded chromosomal abnormality and pathogenic copy number variation}

Karyotyping of the patient indicated the presence of a normal set of chromosomes. Chromosomal array analysis showed a $476 \mathrm{~kb}$ duplication at 14q32.33 (106270453-106746712). This duplication has been classified as a benign polymorphism by the Database of genomic variation (DGV, dgv.tcag.ca) and Phenotype in Humans using Ensembl Resources (DECIPHER, decipher. sanger.ac.uk).

\section{Whole exome sequencing and Sanger sequencing identified two novel mutations in WDR62}

We performed whole exome sequencing of DNA from the proband and her parents. Whole exome sequencing identified two novel heterozygous mutations of the WDR62 gene in the proband: a missense mutation (c. $28 \mathrm{G}>\mathrm{T}$ ) in exon 1 that was inherited from the father, which replaces alanine with serine (p.Ala10Ser) in amino acid 10, and another missense mutation (c.189G $>$ T) in exon 2 , inherited from the mother, which results in substitution of glutamic acid by aspartic acid (p.Glu63Asp) in amino acid 63. We did not find deleterious mutations in any other genes that underlie MCPH. The two mutations were further confirmed by Sanger sequencing (Figure 4). The two mutations were absent in the Human Gene Mutation database (HGMD, www.hgmd.cf.ac.uk/) and MIM. The mutation c. $28 \mathrm{G}>\mathrm{T}$ was not found in BGI's database, which has $~ 30,000$ Chinese Han samples and the frequency of the other mutation, c. $189 \mathrm{G}>\mathrm{T}$ was 0.027 , all the mutations were heterozygous .

(A)

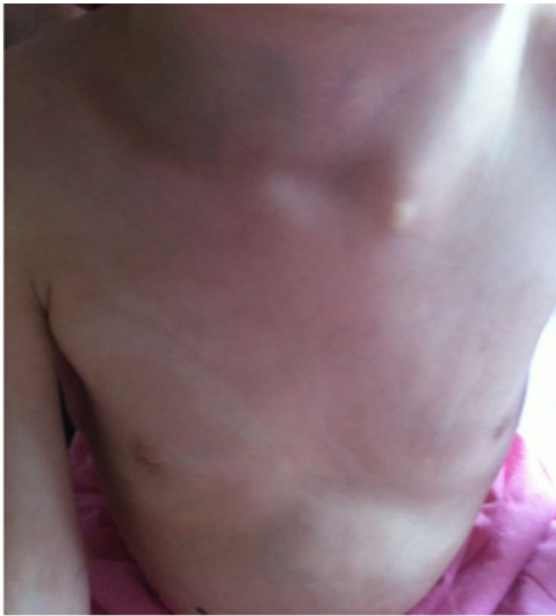

(B)

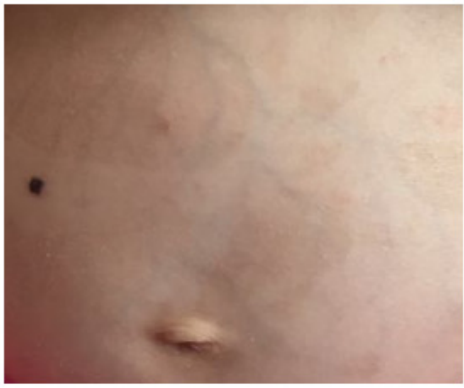

(C)

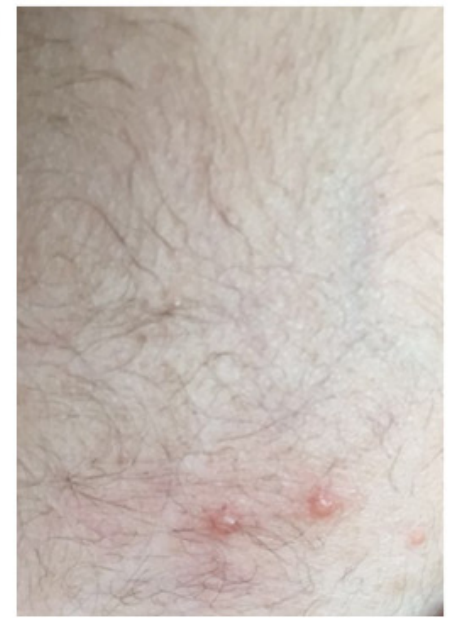

(D)

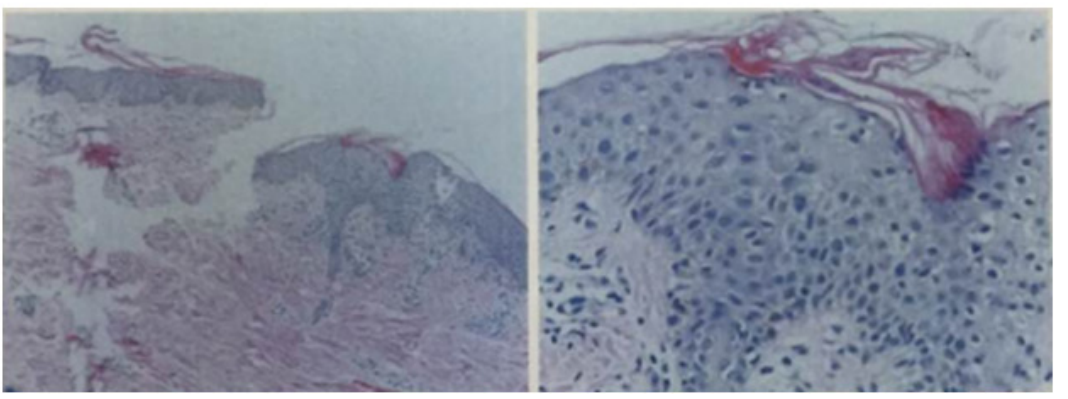

Figure 3: A. and B. Pictures show spattered pigmentation on the trunk. C. Three blisters on the skin. D. HE slides of the skin biopsy showing hyperkeratosis in the skin and porokeratosis. Stratum spinosum thickened slightly with edema in intracellular and intercellular areas. Pigment granules in the basal layer with increased punctate liquefaction degeneration. Few lymphocytes infiltrate beside blood vessel in the shallow corium layer. 


\section{Across-species conservation analysis showed that the two mutations occurred in conserved residues}

Multiple sequence alignment showed that both p.Ala10 and p.Glu63 in WDR62 are evolutionarily highly conserved among different species, indicating their importance in the functions of the wild-type WDR62 protein (Figure 5).

\section{In silico analysis indicated the pathogenic nature of the mutations}

Mutation Taster [10] predicted these two novel mutations to be "disease causing". PolyPhen-2 [11] predicted them to be "probably damaging", and I-MUTANT 2.0 [12] predicted that these mutations would result in "decreasing the stability of the WDR62 protein".

\section{DISCUSSION}

We identified two novel missense mutations in WDR62 gene: a single nucleotide transversion c. $28 \mathrm{G}>\mathrm{T}$ in exon 1 , inherited from the unaffected father, and a single nucleotide transition c. $189 \mathrm{G}>\mathrm{T}$ in exon 2 , inherited from the healthy mother. Mutation Taster predicted these two novel variants to be "disease causing". These two mutations are also predicted to be "decreasing the stability of the WDR62 protein" by I-MUTANT 2.0. Both the mutations (p.10Ala and p.63Glu) affect evolutionarily conserved amino acids. In addition, the replacement of nonpolar/neutral alanine by polar/neutral serine may well affect the protein structure encoded by the wild-type WDR62. According to the guidelines of American College of Medical Genetics and Genomics (ACMG) [13], these two mutations could be classified as "likely pathogenic".

Our array-CGH analysis showed that the patient carried a $476 \mathrm{~kb}$ duplication of 14q32.33 (106270453106746712), which contains the AK128652, KIAA0125, ADAM6 and LINC00226 genes. There has been no report showing that these genes have known functions in brain development or associated with dermatological phenotypes. This duplication has been classified as a "benign polymorphism" by the most widely used CNV databases (DECIPHER and DGV) in clinical genetics practice. We tried to persuade the patient's parents to undergo this analysis. Unfortunately, they rejected consent for another blood sample to be taken for this analysis; therefore the origin of this duplication in the patient is currently unknown.

WDR62 is a scaffold protein of the c-Jun N-terminal kinase (JNK) pathway; it encompasses a WD40 domain, an MKK7 binding domain, and a JNK docking domain.
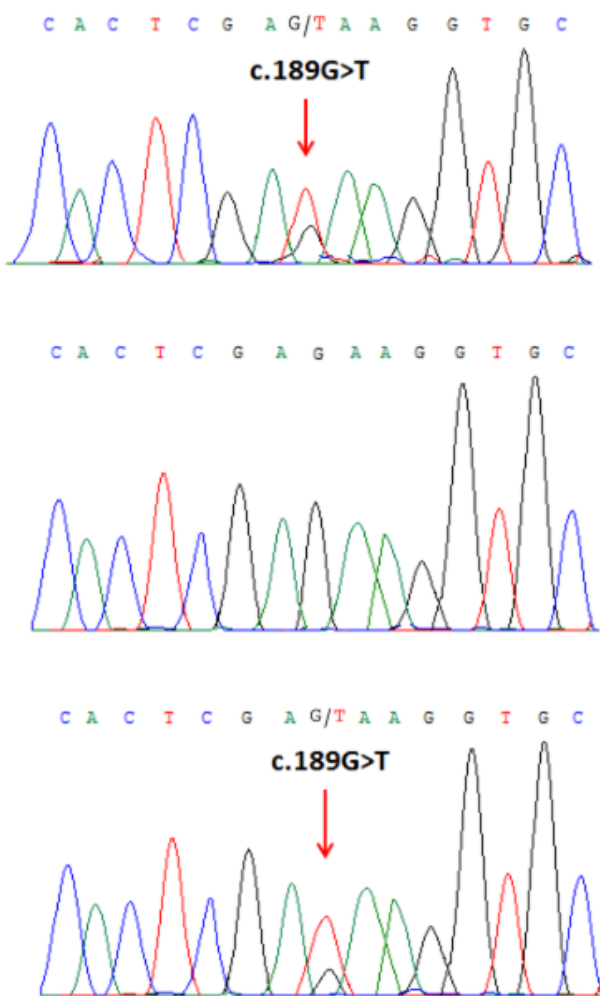

Figure 4: Partial DNA sequences in the WDR62 by Sanger sequencing of the family. Upper line: the proband, middle line: the father, bottom line: the mother. Arrows point to the mutations. The proband inherited both c. $28 \mathrm{G}>\mathrm{T}$ and $\mathrm{c} .189 \mathrm{G}>\mathrm{T}$ mutations. The father carries the c.28G $>$ T mutation, and the mother carries the c.189G $>$ T mutation. 
WDR62 co-localizes to the mitotic spindle poles during early stages of the cell cycle. WDR62 regulates spindle orientation, centrosome integrity, and progression through mitosis [14]. WDR62 is essential for mitotic spindle stabilization during mitosis, and it accumulates at the centrosome or the nucleus in a cell-cycle-dependent manner [14]. Mutant WDR62 proteins fail to localize to the mitotic spindle pole [15]. Presently, 31 mutations of the WDR62 gene have been reported in MCPH2. However, apart from the MCPH2-associated symptoms, our patient also showed hyperkeratosis with typical abnormal skin pigmentation and blisters. Furthermore, in our whole exome sequencing data, we did not identify any deleterious mutations that could cause the reported dermatological

\section{A10S 10 HUMAN MAAVGSGGYARNDAGEKLPSV MOUSE MMAALAAGGYTRSDTIEKLSS MONKEY MAAVGSGGOARNDAGEKLPSV CHIMPANZEE MAAVGSGGYARNDAGEKLPSV ZEBRAFISH MADGAVNF A SMNQSCLTSRA CATTLE MAALGPGGYARNDTVKLPSV DOG MAALGPGGYARNDAVEKLPSG PIG MAALGTGGIVRNDAVEKLPTI E63D HUMAN STASEETVQNRVSL EKVLGITAQNSS MOUSE LAAAPEDTVQNRVTLEKVLGITAQNS MONKEY STASEETVQNRVSLEKVLGITAQNSS CHIMPANZEE STASEETVQNRVSLEKVLGITAQNSS ZEBRAFISH NRVVLEKVLGITASSSSALTCDPNTG CATTLE PAAPEDTVQNRVSLEKVLGITAQNSS DOG STAPEDTVQNRVSLEKVLGITAQNSS PIG TAAPEDAVQNRVSLEKVLGITAQNSS}

Figure 5: Amino acid alignment of the wild-type protein encoded by WDR62. (Homo sapiens) (GenBank Accession: NM_001083961.1) with mouse (Mus musculus) (GenBank Accession: NM_146186.3), rhesus monkey (Macaca mulatta) (GenBank Accession: AFH29290.1), chimpanzee (Pan troglodytes) (GenBank Accession: JAA38944.1), zebrafish (Danio rerio) (GenBank Accession: CM002899.1), cattle (Bos taurus) (GenBank Accession: GK000018.2), dog (Canis lupus familiaris) (GenBank Accession:CM000001.3), and pig (Sus scrofa) (GenBank Accession: CM000817.4). The two boxes show that amino acids 10 and 63 are conserved across these species. 
phenotypes. Our findings in the skin seem to echo two previously reported $\mathrm{MCPH}$ cases, although not $\mathrm{MCPH} 2$ cases, which also presented with dermatological lesions [1]. One possible explanation for the dermatological phenotypes in our patient is that the two novel mutations may cause the loss of the afore mentioned physiological functions of the wild type protein, thereby inducing the dermatological changes. This suggests that WDR62 may play a role in the normal development and maintenance of skin. However, this explanation needs to be further confirmed by other cases and the exact mechanisms need further investigations.

In conclusion, we report the first case of $\mathrm{MCPH} 2$ in a patient who presented with early onset acanthosis and hyperkeratosis, with two underlying novel mutations in the WDR62 gene. Our study not only expands the mutational spectrum for WDR62-associated $\mathrm{MCPH} 2$ but is also valuable for the mutation-based pre- and post-natal screening and genetic diagnosis of $\mathrm{MCPH} 2$.

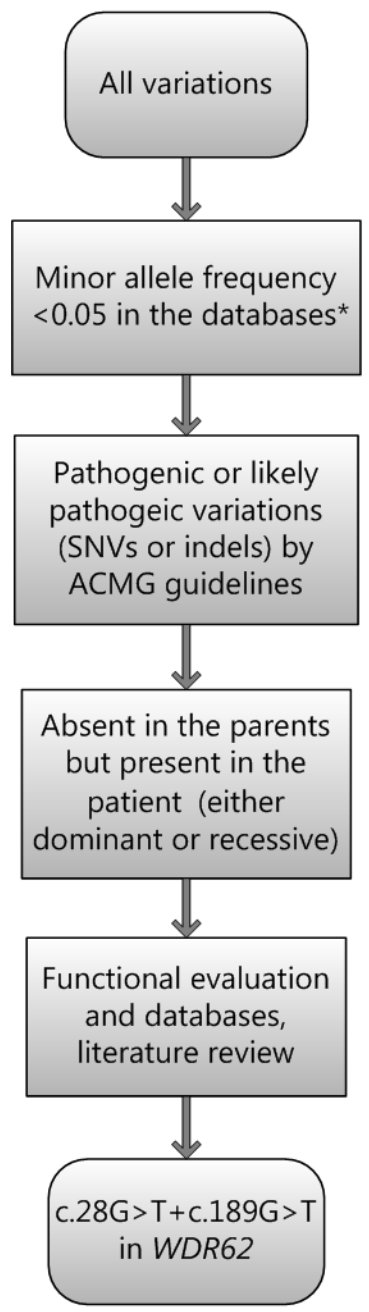

Figure 6: Filtering process for pathogenic mutations in all variations obtained by exome sequencing. *Databases used: dbSNP, Hapmap, 1000 Genomes Project and BGI's in-house database of $\sim 30000$ Chinese people. SNV: single nucleotide variation. Indel: small insertion and deletion.

\section{MATERIALS AND METHODS}

\section{Karyogram and chromosomal array analyses}

Standard G-banding karyotyping was performed. Chromosome microarray analysis was performed using a CytoScan HD array (Affymetrix). The procedures for DNA digestion, ligation, PCR amplification, fragmentation, labeling and hybridization to the array were performed according to the manufacturer's protocols (Affymetrix). The data were analyzed using Chromosome Analysis Suite software version 1.2.2, and the reporting threshold of the copy number was set at $10 \mathrm{~kb}$, with marker count at $\geq 50$, as we previously reported [16].

\section{Whole exome sequencing and mutation selection}

Genomic DNA was extracted from peripheral blood using a QIAamp DNA Blood Mini Kit (Qiagen, Hilden, Germany) according to the manufacturer's instructions. All three family members (parents, proband) were subjected to exome sequencing. Sequences were captured by Agilent SureSelect version 4 (Agilent Technologies, Santa Clara, CA) according to the manufacturer's protocols. The enriched library was sequenced on an Illumina HighSeq2000. The sequencing reads were aligned to GRCh37.p10 using Burrows-Wheeler Aligner software (version 0.59). We then performed local realignment and base quality recalibration of the BurrowsWheeler aligned reads using the GATK IndelRealigner and the GATK BaseRecalibrator, respectively (broadinstitute.org/). Single-nucleotide variants (SNV) and small insertions or deletions (indel) were identified by the GATK UnifiedGenotyper (broadinstitute.org/). Variants were annotated using the Consensus Coding Sequences Database (20130630) at the National Center for Biotechnology Information.

We selected variations obtained from exome sequencing with minor allele frequencies $<0.05$ in any of the following databases (dbSNP, Hapmap, 1000 Genomes Project) and our in-house database for $\sim 30,000$ Chinese Han samples. We also selected pathogenic and likely pathogenic variations according to the ACMG guidelines [13]. We further compared the remaining deleterious variations in the patient with variations carried by her unaffected parents and the gene's functions with the references of OMIM and literature. For the detailed filtering process, please see Figure 6.

\section{Sanger sequencing}

To validate putative mutations, Sanger sequencing was performed. Primers flanking the candidate loci were 
designed based on the reference genomic sequences of the Human Genome from GenBank in NCBI and synthesized by Invitrogen, Shanghai, China. PCR amplification was carried out in an ABI 9700 Thermal Cycler. PCR products were directly sequenced on an ABI PRISM 3730 automated sequencer (Applied Biosystems, Foster City, CA, USA). Sequence data comparisons and analysis were performed by DNASTAR SeqMan (DNASTAR, Madison, Wisconsin, USA).

The compound heterozygous mutations identified through whole exome sequencing were verified through Sanger sequencing using the following primers: F1 5'-ACCCCATGACGCTTAATCAGGC-3', R1 5'-GCAGCTTCTCACCCGGTTCTG-3', F2 5'-AGAAGCTCAGTGTGGGTGTTGAAT-3', and R2 5'-ACAGAGAGGGGGCTATGGAATC-3'. The reference sequence NM_001083961.1 of WDR62 was used.

\section{Bioinformatics analysis}

\section{Evolutionary conservation test}

To understand the evolutionary conservation of the wild type amino acids of the two novel mutations of WDR62 gene, we used ClustalW2 [17] to perform sequence alignment between human, mouse (Mus musculus) (GenBank Accession: NM_146186.3), rhesus monkey (Macaca mulatta) (GenBank Accession: AFH29290.1), chimpanzee (Pan troglodytes) (GenBank Accession: JAA38944.1), zebrafish (Danio rerio) (GenBank Accession: CM002899.1), cattle (Bos taurus) (GenBank Accession: GK000018.2), dog (Canis lupus familiaris) (GenBank Accession:CM000001.3), and pig (Sus scrofa) (GenBank Accession: CM000817.4).The two boxes show that amino acids 10 and 63 are conserved across these species (Figure 5).

\section{In silico prediction}

To analyze the effects of the missense mutations on the functions of the WDR62 protein, we used several bioinformatics programs, viz. MutationTaster [10], PolyPhen2 [11] and I-MUTANT 2.0 [12]. These bioinformatics programs predict the effect of missense mutations on the basis of overall stability, pathogenicity and evolutionary conservation. We used these programs as fundamental and preliminary analyses designed to investigate the molecular mechanism behind the disease phenotype.

\section{ACKNOWLEDGMENTS}

We thank the patient and her parents for participating in this study.

\section{CONFLICTS OF INTEREST}

The authors declare that they have no financial or other conflicts of interest in relation to this research and its publication.

\section{REFERENCES}

1. Belligni EF, Dokal I, Hennekam RC. Prenatal and postnatal growth retardation, microcephaly, developmental delay, and pigmentation abnormalities: Naegeli syndrome, dyskeratosis congenita, poikiloderma Clericuzio type, or separate entity? Eur J Med Genet. 2011;54:231-5.

2. C. Ruth Jamieson, Jean-Pierre Fryns, Jos Jacobs, Gert Matthijs, and Marc J. Abramowicz. Primary Autosomal Recessive Microcephaly: MCPH5 Maps to 1q25-q32. Am J Hum Genet. 2000; 67: 1575-1577.

3. Nicholas AK, Khurshid M, Desir J, Carvalho OP, Cox JJ, Thornton G, Kausar R, Ansar M, Ahmad W, Verloes A, Passemard S, Misson JP, Lindsay S, Gergely F, Dobyns WB, Roberts E, Abramowicz M, Woods CG: WDR62 is associated with the spindle pole and is mutated in human microcephaly. Nat Gen 2010, 42:1010-1014. DOI 10.1038/ ng. 682 .

4. Hofman, M. A. A biometric analysis of brain size in micrencephalics. J. Neurol. 1984; 231: 87-93.

5. Farag HG, Froehler S, Oexle K, Ravindran E, Schindler D, Staab T, Huebner A, Kraemer N, Chen W1, Kaindl AM. Abnormal centrosome and spindle morphology in a patient with autosomal recessive primary microcephaly type 2 due to compound heterozygous WDR62 gene mutation. Orphanet J Rare Dis. 2013 14;8:178.

6. Nicholas AK, Khurshid M, Desir J, Carvalho OP, Cox JJ, Thornton G, Kausar R, Ansar M, Ahmad W, Verloes A, Passemard S, Misson JP, Lindsay S, Gergely F, Dobyns WB, Roberts E, Abramowicz M, Woods CG: WDR62 is associated with the spindle pole and is mutated in human microcephaly. Nat Gen 2010, 42:1010-1014. DOI 10.1038/ ng. 682 .

7. Bilguvar K, Ozturk AK, Louvi A, Kwan KY, Choi M, Tatli B, Yalnizoglu D, Tuysuz B, Caglayan AO, Gokben S, Kaymakcalan H, Barak T, Bakircioglu M, Yasuno K, Ho W, Sanders S, Zhu Y, Yilmaz S, Dincer A, Johnson MH, Bronen RA, Kocer N, Per H, Mane S, Pamir MN, Yalcinkaya C, Kumandas S, Topcu M, Ozmen M, Sestan N, Lifton RP, State MW, Gunel M: Whole-exome sequencing identifies recessive WDR62 mutations in severe brain malformations. Nature 2010, 467:207-210. DOI 10.1038/ nature09327.

8. Yu TW, Mochida GH, Tischfield DJ, Sgaier SK, FloresSarnat L, Sergi CM, Topcu M, McDonaldMT, Barry BJ, Felie JM, Sunu C, Dobyns WB, Folkerth RD, Barkovich AJ, Walsh CA: Mutations in WDR62, encoding a centrosomeassociated protein, cause microcephaly with simplified gyri and abnormal cortical architecture. Nat Gen 2010, 42:1015- 
1020. DOI 10.1038/ng.683.

9. Mahmood S, Ahmad W, Hassan MJ (2011) Autosomal Recessive Primary Microcephaly (MCPH): clinical manifestations, genetic heterogeneity and mutation continuum. Orphanet journal of rare diseases 6: 39.

10. Schwarz JM, Rodelsperger C, Schuelke M, Seelow D: MutationTaster evaluates disease-causing potential of sequence alterations. Nat Methods 2010, 7:575-576. DOI 10.1038/nmeth0810-575.

11. Adzhubei IA, Schmidt S, Peshkin L, Ramensky VE, Gerasimova A, Bork P, Kondrashov AS, Sunyaev SR: A method and server for predicting damaging missense mutations. Nat Methods 2010, 7:248-249. DOI 10.1038/ nmeth0410-248.

12. Capriotti E, Fariselli P, Casadio R. I-Mutant2.0: predicting stability changes upon mutation from the protein sequence or structure. Nucleic Acids Res. 2005 1;33:W306-10.

13. Richards S, Aziz N, Bale S, Bick D, Das S, Gastier-Foster J, Grody WW, Hegde M, Lyon E, Spector E, Voelkerding K, Rehm HL; ACMG Laboratory Quality Assurance Committee. Standards and guidelines for the interpretation of sequence variants: a joint consensus recommendation of the American College of Medical Genetics and Genomics and the Association for Molecular Pathology. Genet Med. 2015; 17:405-24.
14. Bogoyevitch MA, Yeap YY, Qu Z, Ngoei KR, Yip YY, Zhao TT, Heng JI, Ng DC: WD40-repeat protein 62 is a JNK-phosphorylated spindle pole protein required for spindle maintenance and timely mitotic progression. J Cell Sci 2012, 125:5096-5109. DOI 10.1242/jcs. 107326.

15. Nicholas AK, Khurshid M, Desir J, Carvalho OP, Cox JJ, Thornton G, Kausar R, Ansar M, Ahmad W, Verloes A, Passemard S, Misson JP, Lindsay S, Gergely F, Dobyns WB, Roberts E, Abramowicz M, Woods CG: WDR62 is associated with the spindle pole and is mutated in human microcephaly. Nat Gen 2010, 42:1010-1014. DOI 10.1038/ ng.682.

16. Wang Y, Su P, Hu B, Zhu W, Li Q, Yuan P, Li J, Guan X, Li F, Jing X, Li R, Zhang Y, Férec C, Cooper DN, Wang J, Huang D, Chen JM, Wang Y: Characterization of 26 deletion CNVs reveals the frequent occurrence of micro-mutations within the breakpoint-flanking regions and frequent repair of double-strand breaks by templated insertions derived from remote genomic regions.Hum Genet. 2015, 6:589-603. DOI 10.1007/s00439-015-1539-4.

17. Larkin MA, Blackshields G, Brown NP, Chenna R, McGettigan PA, McWilliam H, Valentin F, Wallace IM, Wilm A, Lopez R, Thompson JD, Gibson TJ, Higgins DG. Clustal W and Clustal X version 2.0. Bioinformatics. 2007, $1 ; 23: 2947-8$. 